\title{
Global Stability of a Mumps Transmission Model with Quarantine Measure
}

\author{
Yu-zhen BAI ${ }^{1}$, Xiao-jing WANG ${ }^{1}$, Song-bai GUO ${ }^{1,2, \dagger}$ \\ ${ }^{1}$ School of Science, Beijing University of Civil Engineering and Architecture, Beijing 102616, China \\ $\left({ }^{\dagger}\right.$ E-mail: guosongbai@bucea.edu.cn) \\ ${ }^{2}$ Academy of Mathematics and Systems Science, Chinese Academy of Sciences, Beijing 100190, China
}

\begin{abstract}
In this paper, a model of mumps transmission with quarantine measure is proposed and then the control reproduction number $\mathcal{R}_{c}$ of the model is obtained. This model admits a unique endemic equilibrium $P^{*}$ if and only if $R_{c}>1$, while the disease-free equilibrium $P^{0}$ always exists. By using the technique of constructing Lyapunov functions and the generalized Lyapunov-LaSalle theorem, we first show that the equilibrium $P^{0}$ is globally asymptotically stable (GAS) if $R_{c} \leq 1$; second, we prove that the equilibrium $P^{*}$ is GAS if $R_{c}>1$. Our results reveal that mumps can be eliminated from the community for $\mathcal{R}_{c} \leq 1$ and it will be persistent for $\mathcal{R}_{c}>1$, and quarantine measure can also effectively control the mumps transmission.
\end{abstract}

Keywords mumps transmission model; control reproduction number; quarantine measure; global stability 2000 MR Subject Classification 34D23; 37N25; 92D30

\section{Introduction}

Mumps (epidemic parotitis) is an airborne infectious disease which is resulted from a viral infection of the parotid gland ${ }^{[18]}$. Mumps virus can be spread by sick people sneezing or coughing with tiny droplets of contaminated saliva, which may be breathed in by others, so as to make them be infected ${ }^{[21]}$. In winter and early spring, mumps infections spread very easily ${ }^{[9]}$. Rubin et al. ${ }^{[19]}$ (also see Dittrich et al. ${ }^{[5]}$ ) pointed out that the proportion of asymptomatically infected people was about one third to one half. It was demonstrated by Davis et al. ${ }^{[3]}$ that the exposed period of mumps is 2-3 weeks and it can be diagnosed clinically. Following the discussion of Gutierrez ${ }^{[9]}$, patients are most contagious in the first 1-2 days and the last 5 days of symptoms of mumps, and about $80 \%$ to $90 \%$ of non-immune family contacts become infected. Above all, the current pandemic COVID-19 can be transmitted in the exposed and asymptomatic periods. It is essential to consider the exposed and asymptomatic transmission for traditional infectious disease models. In this paper, as proof of principle, we concerntrate on modeling of mumps which has the exposed and asymptomatic transmission components. Brauer and Castillo-Chavez ${ }^{[1]}$ pointed out the importance of quarantine in a epidemiological framework (also see Erdem et al. ${ }^{[7]}$ ). Kutty et al. ${ }^{[12]}$ also indicated that quarantine was an effective measure to control mumps, and the health care and public health providers in the United States suggested the quarantine period was about five days.

Nowadays, mumps has been reemerging in teenagers and young people around the world. In China, the number of infected people with mumps was more than 300,000 in recent years ${ }^{[13]}$. In

Manuscript received January 21, 2021. Accepted on May 26, 2021.

This work is supported in part by the National Natural Science Foundation of China (Nos. 11901027 and 11871093), the Scientific Research Project of Beijing Municipal Education Commission (No. KM201910016001), the Pyramid Talent Training Project of BUCEA (JDYC20200327), the Bill \& Melinda Gates Foundation (INV005834) and the Fundamental Research Funds for Beijing Universities (X20083).

${ }^{\dagger}$ Corresponding author. 
2004, the quantity of mumps notifications in England and Wales was 16436, and most of them were young adults presenting at universities or colleges ${ }^{[20]}$. During 2006, the United States experienced one multi-state mumps outbreak, reporting 6584 cases, of which 18-24 years old had the most infections, and most of them were college students ${ }^{[4]}$. The United States reported an unusually large number of mumps cases (more than 13800 cases) from the end of 2015 to the end of $2017^{[16]}$. Although the vaccine is the main preventive measure to prevent mumps, it is no longer fully protective for college students because the vaccine was given in childhood. Some countries do not even get the vaccine, such as Japan. Dayan et al. ${ }^{[4]}$ showed that young people who have attended universities or colleges are the main infected group of mumps in recent years.

Epidemiological models, which can help to put forward many practical prevention measures to control the epidemic disease, can be used to better understand the dynamics of infectious diseases. Now, there are a few papers about dynamic models of infectious diseases to describe mumps transmission. In 2002, Jansen et al. ${ }^{[11]}$ considered a discrete model, in which they mainly used a finite-difference method. Li et al. ${ }^{[13]}$ proposed an SVEILR (S: susceptible individuals, V: vaccinated individuals, E: exposed individuals, I: severely infectious individuals, L: mild infections individuals, R: recovered individuals) dynamical transmission model. They mainly considered homogeneous mixing and optimized parameters to depict the infection dynamics of mumps transmission in China. Liu et al. ${ }^{[14]}$ discussed the effects of heterogeneity for spreading mumps, so they established a multi-group SVEIAR (I: symptomatically infected individuals, A: asymptomatically infected individuals) model with exposed and asymptomatic infection stages. They also studied the dynamics property of the model. Qu et al..17] built a non-autonomous SVEILHR (I: mild infectious individuals, L: severe infectious individuals, H: hospitalized individuals) model to understand the epidemic of mumps. They focused on the influence of periodic transmission rates. Based on the researches above, to investigate the effect of exposed individuals and the heterogeneity of infectious rate on the spread and control of mumps, we propose a mathematical model with exposed period and quarantine measure. In our model, we show that the infected people are also contagious in exposed stage, and the infection rates are different among the exposed, symptomatic infectious and asymptomatic infectious individuals.

The remainder of this paper is organized as below. In Sect. 2, the formulations of the model are given. In Sect. 3, we prove that the endemic equilibrium of the model exists if and only if the control reproduction number is greater than one. In Sect. 4, the corresponding necessary and sufficient conditions for global stability of equilibria of the model are presented. Finally, a brief discussion and conclusion is presented.

\section{Model Formulation}

The population is classified into six compartments which are denoted by $S$ : susceptible individuals, $E$ : exposed individuals, $I$ : symptomatically infected individuals, $A$ : asymptomatically infected individuals, $Q$ : quarantined individuals and $R$ : recovered individuals, respectively. Then the dynamic model of mumps transmission is proposed as below:

$$
\begin{aligned}
\dot{S}(t) & =\Lambda-\mu S(t)-\beta S(t)(\delta E(t)+I(t)+m A(t)), \\
\dot{E}(t) & =\beta S(t)(\delta E(t)+I(t)+m A(t))-\mu E(t)-w E(t), \\
\dot{I}(t) & =p w E(t)-\mu I(t)-\varphi I(t)-\gamma I(t), \\
\dot{A}(t) & =(1-p) w E(t)-\mu A(t)-\gamma A(t), \\
\dot{Q}(t) & =\varphi I(t)-\mu Q(t)-\gamma Q(t), \\
\dot{R}(t) & =\gamma I(t)+\gamma A(t)+\gamma Q(t)-\mu R(t) .
\end{aligned}
$$


Here, all parameters of system (2.1) are assumed to be positive. Let $\Lambda$ be the birth rate, $\mu$ be the natural death rate, along with $\beta$ represents the transmission rate; $\delta$ and $m$ indicate regulatory factors for infection probability of the exposed and the asymptomatically infected individuals, respectively. Besides, $w$ stands for transition rate of the exposed individuals to the infected classes; $p$ denotes the transition probability of the symptomatically infected class. Moreover, $\varphi$ is the quarantined rate of the symptomatically infected class and $\gamma$ is the recovery rate.

By the fundamental theorem of ordinary differential equations ${ }^{[15]}$ and $[2$, Proposition 1.1], it is not difficult to obtain the nonnegative cone $\mathbb{R}_{+}^{6}$ is a positive invariant set of system (2.1) which satisfies the well-posedness and the dissipativeness. Hence, we will discuss the global dynamics of system (2.1) in $\mathbb{R}_{+}^{6}$.

\section{Existence of Endemic Equilibrium}

In this section, we shall discuss the global properties of equilibria of system (2.1) by the Lyapunov function method. Obviously, system (2.1) always has a disease-free equilibrium $P^{0}=\left(S^{0}, 0,0,0,0,0\right)$, where $S^{0}=\Lambda / \mu$. For the existence of an endemic equilibrium (i.e., positive equilibrium) $P^{*}=\left(S^{*}, E^{*}, I^{*}, A^{*}, Q^{*}, R^{*}\right)$ of system (2.1), we first give the control reproduction number of system (2.1) by using the approach in [6]:

$$
\mathcal{R}_{c}=\frac{\Lambda \delta \beta}{\mu(w+\mu)}+\frac{\Lambda p w \beta}{\mu(w+\mu) B}+\frac{\Lambda(1-p) w m \beta}{\mu(\mu+w)(\gamma+\mu)},
$$

where $B:=\varphi+\gamma+\mu$. Here, the first term $\Lambda \delta \beta / \mu(w+\mu)$ can be interpreted as the average number of new infections generated through exposed individuals. The second term $\Lambda p w \beta / \mu(w+\mu) B$ can be interpreted as the average number of new infections generated through symptomatically infected individuals. The third term $\Lambda(1-p) w m \beta / \mu(w+\mu)(\gamma+\mu)$ can be interpreted as the average number of new infections generated through asymptomatically infected individuals.

Lemma 3.1. System (2.1) has a unique endemic equilibrium $P^{*}$ if and only if $\mathcal{R}_{c}>1$ holds.

Proof. By the equilibrium equations of system (2.1), we can get

$$
\begin{aligned}
S & =\frac{\Lambda(\gamma+\mu) B}{\beta p w(\gamma+\mu) E+[(\beta \delta E+\mu)(\gamma+\mu)+\beta(1-p) w m E] B}=\frac{\Lambda-(\mu+w) E}{\mu} \\
I & =\frac{p w E}{B}, A=\frac{(1-p) w E}{\gamma+\mu}, Q=\frac{\varphi p w E}{(\gamma+\mu) B}, R=\frac{\gamma \varphi p w E}{\mu(\gamma+\mu) B}+\frac{\gamma p w E}{\mu B}+\frac{(1-p) w \gamma E}{\mu(\gamma+\mu)} .
\end{aligned}
$$

From equation (3.1), it follows

$$
E(a E-b)=0,
$$

where

$$
a=\frac{\mu(\gamma+\mu)(w+\mu)^{2} B \mathcal{R}_{c}}{\Lambda}>0, \quad b=\mu(\gamma+\mu)(w+\mu) B\left(\mathcal{R}_{c}-1\right) .
$$

Consequently, system (2.1) exists a unique endemic equilibrium $P^{*} \gg \mathbf{0}$ which is equivalent to $0<E^{*}=b / a<\Lambda /(w+\mu)$. Obviously, equation (3.2) admits a unique positive root $E^{*}<\Lambda /(w+\mu)$ if and only if $\mathcal{R}_{c}>1$. Therefore, the conclusion of Lemma 3.1 holds.

\section{Global Stability}

In this section, we will obtain the global stability results of equilibria $P^{0}$ and $P^{*}$. Consider the following differential system

$$
\dot{x}(t)=g(x),
$$


where $g: \mathbb{R}^{n} \rightarrow \mathbb{R}^{n}$ is continuous, and a solution of system (4.1) with any $\phi \in \mathbb{R}^{n}$ exists uniquely, which depends continuously upon the initial data. Let $X$ be a subset of $\mathbb{R}^{n}$ and the solution $x(t)$ of system (4.1) through any $\phi \in X$ exist on $[0, \infty)$ and $X$ be positively invariant for system (4.1). Let $\omega(\phi)$ be the $\omega$-limit set of $\phi$ with respect to system (4.1) and $P \in X$ an equilibrium of system (4.1). To prove our main results, we need the following two lemmas (also see [8]). In fact, the following lemma is also a corollary of the Lyapunov-LaSalle theorem.

Lemma 4.1 (Generalized Lyapunov-LaSalle theorem). Let the solution $x(t)$ of system (4.1) through any $\phi \in X$ be bounded. If there is $T=T(\phi) \geq 0$ such that $V$ is a Lyapunov function on $\mathcal{O}_{T}(\phi):=\{x(t): t \geq T\}$ (that is, $V$ is continuous on $\overline{\mathcal{O}_{T}(\phi)}$ (the closure of $\mathcal{O}_{T}(\phi)$ ) and $\dot{V} \leq 0$ on $\left.\mathcal{O}_{T}(\phi)\right)$, then $\dot{V}=0$ on $\omega(\phi)$.

Lemma 4.2. Let $V \in C\left(\mathbb{R}^{n}, \mathbb{R}_{+}\right)$be a positive definite function with respect to the equilibrium $P$. If there exists $T>0$ which is independent of $\phi$ such that $V$ is continuous on $\mathcal{O}_{T}(\phi)$ and $u_{T}(X)$, respectively, and for any $\psi \in \mathcal{O}_{T}(\phi)$,

$$
\dot{V}(\psi) \leq 0,
$$

then the equilibrium $P$ is uniformly stable.

For the disease-free equilibrium $P^{0}$, we have the following theorem.

Theorem 4.3. The disease-free equilibrium $P^{0}$ is GAS if and only if $\mathcal{R}_{c} \leq 1$ holds.

Proof. It easily follows from [6, Theorem 2] that the equilibrium $P^{0}$ is unstable if $\mathcal{R}_{c}>1$. We thus only need to prove sufficiency. We shall first prove that the equilibrium $P^{0}$ is globally attractive. Assuming

$$
\mathcal{U}(t):=(S(t), E(t), I(t), A(t), Q(t), R(t))
$$

is the solution of system (2.1) through any $\phi:=\left(\phi_{1}, \phi_{2}, \phi_{3}, \phi_{4}, \phi_{5}, \phi_{6}\right) \in \mathbb{R}_{+}^{6}$. Clearly, $\mathcal{U}(t)$ is bounded and $S(t)>0$ for $t>0$. Let we define a function $V$ on $\Omega=\left\{\phi \in \mathbb{R}_{+}^{6}: \phi_{1}>0\right\}$ as follows,

$$
V(\phi)=\phi_{1}-S^{0}-S^{0} \ln \frac{\phi_{1}}{S^{0}}+\phi_{2}+\frac{\beta S^{0}}{B} \phi_{3}+\frac{\beta S^{0} m}{\gamma+\mu} \phi_{4} .
$$

It can be easily seen that $V$ is a continuous function on $\Omega \subseteq \mathbb{R}_{+}^{6}$. The derivative of $V$ along $\mathcal{U}(t)$ for $t>0$ can be expressed by

$$
\begin{aligned}
\dot{V}(\mathcal{U}(t))= & \left(1-\frac{S^{0}}{S(t)}\right) \dot{S}(t)+\dot{E}(t)+\frac{\beta S^{0}}{B} \dot{I}(t)+\frac{\beta S^{0} m}{\gamma+\mu} \dot{A}(t) \\
= & \Lambda-\mu S(t)-\frac{S^{0} \Lambda}{S(t)}+\mu S^{0}+\beta S^{0} \delta E(t)-(w+\mu) E(t) \\
& +\frac{\beta S^{0} p w E(t)}{B}+\frac{\beta S^{0} m(1-p) w E(t)}{\gamma+\mu} \\
= & -\mu \frac{\left(S^{0}-S(t)\right)^{2}}{S(t)}+E(t)(w+\mu)\left(\mathcal{R}_{c}-1\right) \\
\leq & 0 .
\end{aligned}
$$

By (4.3) and (4.4), $S(t)$ is persistent, that is, there is some $\epsilon=\epsilon(\phi)>0$ such that $\lim _{\inf } \rightarrow \infty S(t)$ $>\epsilon$. Thus, we have $\omega(\phi) \subseteq \Omega$. Hence if $\mathcal{R}_{c} \leq 1, V$ is a Lyapunov function on $\{\mathcal{U}(t): t \geq 1\} \subseteq \Omega$. From Lemma 4.1, we know that for any $\psi \in \omega(\phi), \dot{V}(\psi)=0$. Next, to prove the equilibrium $P^{0}$ is globally attractive, we only verify $\omega(\phi)=\left\{P^{0}\right\}$.

Assume that $\mathcal{U}(t)=(S(t), E(t), I(t), A(t), Q(t), R(t))$ is the solution of system (2.1) through any $\psi \in \omega(\phi)$. Then by the invariance of $\omega(\phi)$, we have that $\mathcal{U}(t) \in \omega(\phi)$ for all $t \in \mathbb{R}$. Because 
$\dot{V}(\mathcal{U}(t))=0$ for all $t \in \mathbb{R}$ and (4.4), it follows $S(t)=S^{0}$ for all $t \in \mathbb{R}$. Next, by the first equation of system $(2.1)$, it holds $I(t)=A(t)=E(t)=0$ for all $t \in \mathbb{R}$. From the fifth and the sixth equations of system (2.1) and the invariance of $\omega(\phi)$, it holds $Q(t)=R(t)=0$ for all $t \in \mathbb{R}$. Thus, we have $\omega(\phi)=\left\{P^{0}\right\}$.

In the following, we only prove the equilibrium $P^{0}$ is stable.

Considering that the first four equations of system (2.1) can constitute an independent subsystem, denoted as system (I). Clearly, the nonnegative cone $\mathbb{R}_{+}^{4}$ is positive invariant for system (I). It follows from (4.3), (4.4) and Lemma 4.2 that the disease-free equilibrium $X^{0}=$ $\left(S^{0}, 0,0,0\right)$ of system (I) is stable. Let $l:=\min \left\{\frac{\gamma+\mu}{\varphi}, \frac{\mu}{\gamma}, 1\right\}$. By the definition of stability of an equilibrium, we have that for any $\varepsilon>0$, there can be found $\delta \leq \varepsilon l / 5$ such that for any $\psi \in \mathbb{R}_{+}^{4}$ and $\left\|\psi-X^{0}\right\|<\delta$, it holds

$$
\left\|X(t)-X^{0}\right\|<\frac{\varepsilon l^{2}}{5}, \quad \text { for all } t \geq 0
$$

where $X(t)$ is the solution of system (I) through $\psi$. The solutions of the fifth and the sixth equations of system (2.1) with any $\phi \in \mathbb{R}_{+}^{6}$ can be obtained as follows:

$$
\begin{aligned}
& Q(t)=\phi_{5} e^{-(\gamma+\mu) t}+\varphi e^{-(\gamma+\mu) t} \int_{0}^{t} I(s) e^{(\gamma+\mu) s} d s \\
& R(t)=\phi_{6} e^{-\mu t}+\gamma e^{-\mu t} \int_{0}^{t} Q(s) e^{\mu s} d s+\gamma e^{-\mu t} \int_{0}^{t} I(s) e^{\mu s} d s+\gamma e^{-\mu t} \int_{0}^{t} A(s) e^{\mu s} d s .
\end{aligned}
$$

For any $\phi \in \mathbb{R}_{+}^{6}$ and $\left\|\phi-P^{0}\right\|<\delta$, we have that for any $t \geq 0$,

$$
Q(t)<\frac{\varepsilon l}{5} e^{-(\gamma+\mu) t}+\varphi \frac{\varepsilon l^{2}}{5} e^{-(\gamma+\mu) t} \int_{0}^{t} e^{(\gamma+\mu) s} d s \leq \frac{\varepsilon l}{5} e^{-(\gamma+\mu) t}+\varphi \frac{\varepsilon l}{5} \frac{\gamma+\mu}{\varphi} \frac{1-e^{-(\gamma+\mu) t}}{\gamma+\mu}=\frac{\varepsilon l}{5}
$$

and

$$
R(t)<\frac{3 \varepsilon}{5} e^{-\mu t}+\gamma \frac{\varepsilon}{5} \frac{\mu}{\gamma} \frac{1-e^{-\mu t}}{\mu}+\gamma \frac{\varepsilon}{5} \frac{\mu}{\gamma} \frac{1-e^{-\mu t}}{\mu}+\gamma \frac{\varepsilon}{5} \frac{\mu}{\gamma} \frac{1-e^{-\mu t}}{\mu}=\frac{3 \varepsilon}{5} .
$$

As a consequence,

$$
\left\|\mathcal{U}(t)-P^{0}\right\| \leq\left\|X(t)-X^{0}\right\|+Q(t)+R(t)<\frac{\varepsilon l^{2}}{5}+\frac{\varepsilon l}{5}+\frac{3 \varepsilon}{5} \leq \varepsilon .
$$

Thus, the equilibrium $P^{0}$ is stable.

The following conclusion is about the global property of the equilibrium $P^{*}$.

Theorem 4.4. The endemic equilibrium $P^{*}$ is $G A S$ if and only if $\mathcal{R}_{c}>1$ in $\Gamma=\left\{\phi \in \mathbb{R}_{+}^{6}\right.$ : $\left.\phi_{2}>0\right\}$.

Proof. By Lemma 3.1, we only need to prove sufficiency. Clearly, the set $\Gamma$ is positively invariant for system (2.1). We first show that the equilibrium $P^{*}$ is globally attractive. Assuming $\mathcal{U}(t)=(S(t), E(t), I(t), A(t), Q(t), R(t))$ is the solution of system (2.1) through any $\phi \in \Gamma$. It is easy to find that $\mathcal{U}(t)$ is bounded and $\mathcal{U}(t) \gg \mathbf{0}$ for $t>0$. To show that the equilibrium $P^{*}$ is globally attractive, we only prove $\omega(\phi)=\left\{P^{*}\right\}$. Now, define a function $V$ on $\operatorname{Int}\left(\mathbb{R}_{+}^{6}\right)$ (the interior of $\mathbb{R}_{+}^{6}$ ) as follows,

$$
V(\phi)=S^{*} h\left(\frac{\phi_{1}}{S^{*}}\right)+E^{*} h\left(\frac{\phi_{2}}{E^{*}}\right)+\frac{\beta S^{*} I^{*}}{p w E^{*}} I^{*} h\left(\frac{\phi_{3}}{I^{*}}\right)+\frac{\beta m S^{*} A^{*}}{(1-p) w E^{*}} A^{*} h\left(\frac{\phi_{4}}{A^{*}}\right),
$$


where $h(\vartheta)=\vartheta-1-\ln \vartheta, \vartheta>0$. For $t>0$, the derivative of $V$ along $\mathcal{U}(t)\left(\in \operatorname{Int}\left(\mathbb{R}_{+}^{6}\right)\right)$ can be obtained by

$$
\begin{aligned}
\dot{V}(\mathcal{U}(t))= & \left(1-\frac{S^{*}}{S(t)}\right) \dot{S}(t)+\left(1-\frac{E^{*}}{E(t)}\right) \dot{E}(t)+\frac{\beta S^{*} I^{*}}{p w E^{*}}\left(1-\frac{I^{*}}{I(t)}\right) \dot{I}(t) \\
& +\frac{\beta m S^{*} A^{*}}{(1-p) w E^{*}}\left(1-\frac{A^{*}}{A(t)}\right) \dot{A}(t) \\
= & -\Lambda\left(h\left(\frac{S^{*}}{S(t)}\right)+h\left(\frac{S(t)}{S^{*}}\right)\right)+\left(\beta S^{*} I^{*}+\beta m S^{*} A^{*}+\beta \delta S^{*} E^{*}\right) h\left(\frac{S(t)}{S^{*}}\right) \\
& -\beta S^{*} I^{*}\left(\frac{E(t) I^{*}}{E^{*} I(t)}+\frac{S(t) I(t) E^{*}}{S^{*} I^{*} E(t)}-\ln \frac{S(t)}{S^{*}}-2\right) \\
& -\beta m S^{*} A^{*}\left(\frac{E(t) A^{*}}{E^{*} A(t)}+\frac{S(t) A(t) E^{*}}{S^{*} A^{*} E(t)}-\ln \frac{S(t)}{S^{*}}-2\right) \\
& -\beta \delta S^{*} E^{*}\left(\frac{S(t)}{S^{*}}-1-\ln \frac{S(t)}{S^{*}}\right) \\
= & -\Lambda h\left(\frac{S^{*}}{S(t)}\right)-\left(\mu S^{*}+\beta \delta S^{*} E^{*}\right) h\left(\frac{S(t)}{S^{*}}\right) \\
& -\beta S^{*} I^{*}\left(h\left(\frac{E(t) I^{*}}{E^{*} I(t)}\right)+h\left(\frac{S(t) I(t) E^{*}}{S^{*} I^{*} E(t)}\right)\right) \\
& -\beta m S^{*} A^{*}\left(h\left(\frac{E(t) A^{*}}{E^{*} A(t)}\right)+h\left(\frac{S(t) A(t) E^{*}}{S^{*} A^{*} E(t)}\right)\right) \leq 0,
\end{aligned}
$$

where the equations of the endemic equilibrium $P^{*}: \mu=\Lambda / S^{*}-\beta\left(I^{*}+m A^{*}+\delta E^{*}\right)$,

$$
w+\mu=\beta \frac{S^{*} I^{*}}{E^{*}}+\beta m \frac{S^{*} A^{*}}{E^{*}}+\beta \delta S^{*}, \quad \mu+\varphi=p w \frac{E^{*}}{I^{*}}-\gamma, \quad \gamma+\mu=(1-p) w \frac{E^{*}}{A^{*}}
$$

are used.

By virtue of (4.6) and (4.7), we can prove that $\omega(\phi) \subseteq \operatorname{Int}\left(\mathbb{R}_{+}^{6}\right)$. Accordingly, if $\mathcal{R}_{c}>1$, $V$ is a Lyapunov function on $\{\mathcal{U}(t): t \geq 1\} \subseteq \operatorname{Int}\left(\mathbb{R}_{+}^{6}\right)$. It follows from Lemma 4.1 that for any $\psi=\left(\psi_{1}, \psi_{2}, \psi_{3}, \psi_{4}, \psi_{5}, \psi_{6}\right) \in \omega(\phi)$, there hold $\psi_{1}=S^{*}, \psi_{2} I^{*}=E^{*} \psi_{3}, \psi_{2} A^{*}=E^{*} \psi_{4}$. Assume that $\mathcal{U}(t)=(S(t), E(t), I(t), A(t), Q(t), R(t))$ is the solution of system (2.1) through any $\psi \in \omega(\phi)$. Then it follows from the invariance of $\omega(\phi)$ that $\mathcal{U}(t) \in \omega(\phi)$ for all $t \in \mathbb{R}$. In consequence, $S(t)=S^{*}, E^{*} I(t)=E(t) I^{*}$ and $E(t) A^{*}=E^{*} A(t)$ for all $t \in \mathbb{R}$. Hence, we have

$$
E^{*} \dot{I}(t)=E^{*} p w E(t)-B E^{*} I(t)=E^{*} p w E(t)-B E(t) I^{*}=E(t)\left(p w E^{*}-B I^{*}\right)=0,
$$

which hints that $I(t)$ is a constant function on $\mathbb{R}$. The invariance of $\omega(\phi)$ and the system (2.1) yield that the four functions $E(t), A(t), Q(t)$ and $R(t)$ are all constants. In consequence, $\mathcal{U}(t)(\gg \mathbf{0})$ is a positive equilibrium of system (2.1). Consider that the positive equilibrium is unique, we thus have $\mathcal{U}(t)=P^{*}$ for all $t \in \mathbb{R}$. Therefore, we can obtain $\omega(\phi)=\left\{P^{*}\right\}$.

The following proof shows that the equilibrium $P^{*}$ is stable.

By a similar argument as in the proof of Theorem 3.1, we can get that for any $\varepsilon>0$, there can be found $\delta \leq \varepsilon l / 5$ such that for any $\psi \in \Gamma_{0}=\left\{\psi=\left(\psi_{1}, \psi_{2}, \psi_{3}, \psi_{4}\right) \in \mathbb{R}_{+}^{4}: \psi_{2}>0\right\}$ and $\left\|\psi-X^{*}\right\|<\delta$, it follows

$$
\left\|X(t)-X^{*}\right\|<\frac{\varepsilon l^{2}}{5} \quad \text { for all } t \geq 0
$$

where $X^{*}=\left(S^{*}, E^{*}, I^{*}, A^{*}\right)$ is the endemic equilibrium of system (I). Now, we consider the solutions $Q(t)$ and $R(t)$ of the fifth and the sixth equations of system (2.1) with any $\phi \in \Gamma$ as 
obtained in (4.5). For any $\phi \in \Gamma$ and $\left\|\phi-P^{*}\right\|<\delta$, we have that for any $t \geq 0$,

$$
\begin{aligned}
\left\|Q(t)-Q^{*}\right\| & <\frac{\varepsilon l}{5} e^{-(\gamma+\mu) t}+\varphi \frac{\varepsilon l^{2}}{5} e^{-(\gamma+\mu) t} \int_{0}^{t} e^{(\gamma+\mu) s} d s \\
& \leq \frac{\varepsilon l}{5} e^{-(\gamma+\mu) t}+\varphi \frac{\varepsilon l}{5} \frac{\gamma+\mu}{\varphi} \frac{1-e^{-(\gamma+\mu) t}}{\gamma+\mu} \\
& =\frac{\varepsilon l}{5}
\end{aligned}
$$

and

$$
\begin{aligned}
\left\|R(t)-R^{*}\right\| & <\frac{\varepsilon}{5} e^{-\mu t}+\gamma \frac{\varepsilon l}{5} \frac{1-e^{-\mu t}}{\mu}+\gamma \frac{\varepsilon l}{5} \frac{1-e^{-\mu t}}{\mu}+\gamma \frac{\varepsilon l}{5} \frac{1-e^{-\mu t}}{\mu} \\
& <\frac{3 \varepsilon}{5} e^{-\mu t}+\gamma \frac{\varepsilon}{5} \frac{\mu}{\gamma} \frac{1-e^{-\mu t}}{\mu}+\gamma \frac{\varepsilon}{5} \frac{\mu}{\gamma} \frac{1-e^{-\mu t}}{\mu}+\gamma \frac{\varepsilon}{5} \frac{\mu}{\gamma} \frac{1-e^{-\mu t}}{\mu} \\
& =\frac{3 \varepsilon}{5}
\end{aligned}
$$

where

$$
\begin{aligned}
& Q^{*}=Q^{*} e^{-(\gamma+\mu) t}+\varphi e^{-(\gamma+\mu) t} \int_{0}^{t} I^{*} e^{(\gamma+\mu) s} d s \\
& R^{*}=R^{*} e^{-\mu t}+\gamma e^{-\mu t} \int_{0}^{t} Q^{*} e^{\mu s} d s+\gamma e^{-\mu t} \int_{0}^{t} I^{*} e^{\mu s} d s+\gamma e^{-\mu t} \int_{0}^{t} A^{*} e^{\mu s} d s .
\end{aligned}
$$

In consequence,

$$
\left\|\mathcal{U}(t)-P^{*}\right\| \leq\left\|X(t)-X^{*}\right\|+\left\|Q(t)-Q^{*}\right\|+\left\|R(t)-R^{*}\right\|<\frac{\varepsilon l^{2}}{5}+\frac{\varepsilon l}{5}+\frac{3 \varepsilon}{5} \leq \varepsilon .
$$

Therefore, the equilibrium $P^{*}$ is stable. Thus, the equilibrium $P^{*}$ is GAS if $\mathcal{R}_{c}>1$.

\section{Discussion and Conclusion}

It is not easy to analyse the stability of the equilibrium $P^{*}$ by Lyapunov indirect method, i.e., the stability of the equilibrium $P^{*}$ is determined by analyzing the eigenvalues of characteristic equation of the corresponding linearized system of system (2.1) at the equilibrium $P^{*}$. Consequently, we gain the stability of equilibria of system (2.1) by using Lemma 4.2 with the original details. Consider that Lyapunov functions (4.3) and (4.6) do not meet the condition of the classical Lyapunov-LaSalle theorem (see [10, Theorem X.1.3]), and the largest invariant set $M \subset E=\left\{\phi \in \mathbb{R}_{+}^{6}: \dot{V}(\phi)=0\right\}$ in the theorem may be unbounded. Thus, we introduce the generalized Lyapunov-LaSalle theorem (see Lemma 4.1) to obtain global stability of equilibria of system (2.1).

In this study, we have proposed and analyzed an SEIAQR model of mumps transmission. The global dynamics of the model has been discussed in terms of the control reproduction number $\mathcal{R}_{c}$. The mathematical results have shown that the equilibrium $P^{0}$ is GAS in $\mathbb{R}_{+}^{6}$ if and only if $\mathcal{R}_{c} \leq 1$ holds; the equilibrium $P^{*}$ is GAS in $\Gamma$ if and only if $\mathcal{R}_{c}>1$ holds. These mean that mumps can be eliminated from the community when $\mathcal{R}_{c} \leq 1$ and it will be persistent when $\mathcal{R}_{c}>1$. Besides, it can also be used to study current COVID-19 pandemic by our modeling ideas.

Note that the control reproduction number $\mathcal{R}_{c}$ is an increasing function with respect to the parameters $\delta$ and $m$ respectively, which are the proportion of exposed individuals and 
asymptomatically infected individuals respectively who can transmit the disease to susceptible individuals. If all other parameters are fixed, the dynamics of model (2.1) can changed significantly as parameters $\delta$ and $m$ increase, once $\mathcal{R}_{c}$ increases from less than 1 to more than 1. In addtion, $\mathcal{R}_{c}$ is a decreasing function with respect to the quarantined rate $\varphi$, which means that $\mathcal{R}_{c}$ will decrease with $\varphi$ increasing. Thus, we can more effectively control the mumps transmission by improving the quarantined rate.

Acknowledgments. The authors would like to thank Prof. Jing-an Cui for his valuable suggestions.

\section{References}

[1] Brauer, F., Castillo-Chavez, C. Mathematical Models in Population Biology and Epidemiology. 2nd ed. Springer-Verlag, New York, 2012, 377-380.

[2] Chepyzhov, V.V., Vishik, M.I. Attractors for Equations of Mathematical Physics. American Mathematical Society, Providence, 2002.

[3] Davis, N.F., McGuire, B.B., Mahon, J.A., et al. The increasing incidence of mumps orchitis: a comprehensive review. BJU International, 105: 1060-1065 (2010)

[4] Dayan, G.H., Quinlisk, M.P., Parker, A.A., et al. Recent resurgence of mumps in the United States. New England Journal of Medicine, 358: 1580-1589 (2008)

[5] Dittrich, S., Hahné, S., van Lier, A., et al. Assessment of serological evidence for mumps virus infection in vaccinated children. Vaccine, 29: 9271-9275 (2011)

[6] van den Driessche, P., Watmough, J. Reproduction numbers and sub-threshold endemic equilibria for compartmental models of disease transmission. Mathematical Biosciences, 180: 29-48 (2002)

[7] Erdem, M., Safan, M., Castillo-Chavez, C. Mathematical analysis of an SIQR influenza model with imperfect quarantine. Bulletin of Mathematical Biology, 79: 1612-1636 (2017)

[8] Guo, S., Ma, W. Remarks on a variant of Lyapunov-LaSalle theorem. Mathematical Biosciences and Engineering, 16: 1056-1066 (2019)

[9] Gutierrez, K.M. Mumps virus, in: Long, S.S., Pickering, L.K., Prober, C.G. (eds.), Principles and Practice of Pediatric Infectious Diseases. 3rd ed. Elsevier Inc., Philadelphia, 2008, 1108-1112.

[10] Hale, J.K. Ordinary Differential Equations. 2nd ed. Robert E. Krieger Publishing Company, Malabar, Florida, 1980

[11] Jansen, H., Twizell, E.H. An unconditionally convergent discretization of the SEIR model. Mathematics and Computers in Simulation, 58: 147-158 (2002)

[12] Kutty, P.K., Kyaw, M.H., Dayan, G.H., et al. Guidance for isolation precautions for mumps in the United States: A review of the ccientific basis for policy change. Clinical Infectious Diseases, 50: 1619-1628 (2010)

[13] Li, Y., Liu, X., Wang, L. Modeling the transmission dynamics and control of mumps in mainland China. International Journal of Environmental Research Public Health, 15: 33 (2018)

[14] Liu, Z., Hu, J., Wang, L. Modelling and analysis of global resurgence of mumps: A multi-group epidemic model with asymptomatic infection, general vaccinated and exposed distributions. Nonlinear Analysis: Real World Applications, 37: 137-161 (2017)

[15] Ma, Z., Zhou, Y., Li, C. Qualitative and Stability Methods of Ordinary Differential Equations. 2nd ed. Science Press, Beijing, 2015 (in Chinese).

[16] McNall, R.J., Wharton, A.K., Anderson, R., et al. Genetic characterization of mumps viruses associated with the resurgence of mumps in the United States: 2015-2017. Virus Research, 281: 197935 (2020)

[17] Qu, Q., Fang, C., Zhang, L., et al. A mumps model with seasonality in China. Infectious Disease Modelling, 2: 1-11 (2017)

[18] Rima, B.K. Mumps Virus, in: Granoff, A., Webster, R.G. (eds.), Encyclopedia of Virology. 2nd ed. Academic Press, San Diego, 1999, 988-994.

[19] Rubin, S., Eckhaus, M., Rennick, L.J., et al. Molecular biology, pathogenesis and pathology of mumps virus. Journal of Pathology, 235: 242-252 (2015)

[20] Savage, E., Ramsay, M., White, J., et al. Mumps outbreaks across England and Wales in 2004: observational study. British Medical Journal, 330: 1119-1120 (2005)

[21] World Health, O. Mumps. https://www.who.int/immunization/diseases/mumps/en, 3 November 2016. 